Yves Demazeau - Takayuki Ito

Javier Bajo • Maria José Escalona (Eds.)

\title{
Advances in Practical
} Applications of Scalable Multi-agent Systems

\section{The PAAMS Collection}

14th International Conference, PAAMS 2016

Sevilla, Spain, June 1-3, 2016 Proceedings

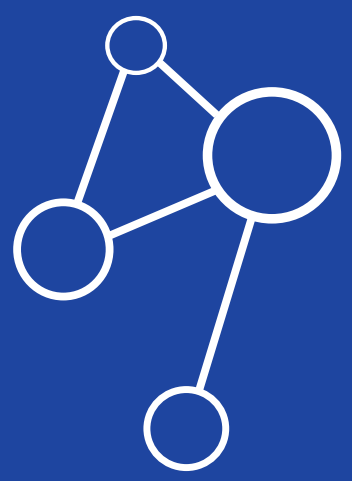

Springer 


\section{Lecture Notes in Artificial Intelligence}

Subseries of Lecture Notes in Computer Science

\section{LNAI Series Editors}

Randy Goebel

University of Alberta, Edmonton, Canada

Yuzuru Tanaka

Hokkaido University, Sapporo, Japan

Wolfgang Wahlster

DFKI and Saarland University, Saarbrücken, Germany

LNAI Founding Series Editor

Joerg Siekmann

DFKI and Saarland University, Saarbrücken, Germany 
More information about this series at http://www.springer.com/series/1244 
Yves Demazeau · Takayuki Ito

Javier Bajo · Maria José Escalona (Eds.)

Advances in Practical

Applications of Scalable

Multi-agent Systems

The PAAMS Collection

14th International Conference, PAAMS 2016

Sevilla, Spain, June 1-3, 2016

Proceedings

黛 Springer 


\section{Editors}

Yves Demazeau

Laboratoire d'Informatique de Grenoble

Centre National de la Recherche Scientifique

Grenoble

France

Takayuki Ito

Department of Computer Science and Engineering

Nagoya Institute of Technology

Nagoya

Japan
Javier Bajo

Departamento de Inteligencia Artificial

Universidad Politécnica de Madrid

Boadilla del Monte, Madrid

Spain

Maria José Escalona

ETS Ingenieria Informatica

Universidad de Sevilla

Sevilla

Spain

ISSN 0302-9743

ISSN 1611-3349 (electronic)

Lecture Notes in Artificial Intelligence

ISBN 978-3-319-39323-0

ISBN 978-3-319-39324-7 (eBook)

DOI 10.1007/978-3-319-39324-7

Library of Congress Control Number: 2016939381

LNCS Sublibrary: SL7 - Artificial Intelligence

(C) Springer International Publishing Switzerland 2016

This work is subject to copyright. All rights are reserved by the Publisher, whether the whole or part of the material is concerned, specifically the rights of translation, reprinting, reuse of illustrations, recitation, broadcasting, reproduction on microfilms or in any other physical way, and transmission or information storage and retrieval, electronic adaptation, computer software, or by similar or dissimilar methodology now known or hereafter developed.

The use of general descriptive names, registered names, trademarks, service marks, etc. in this publication does not imply, even in the absence of a specific statement, that such names are exempt from the relevant protective laws and regulations and therefore free for general use.

The publisher, the authors and the editors are safe to assume that the advice and information in this book are believed to be true and accurate at the date of publication. Neither the publisher nor the authors or the editors give a warranty, express or implied, with respect to the material contained herein or for any errors or omissions that may have been made.

Printed on acid-free paper

This Springer imprint is published by Springer Nature

The registered company is Springer International Publishing AG Switzerland 


\title{
An Agent-Based Model of Labor Market Participation with Health Shocks
}

\author{
Alessandro Moro ${ }^{(凶)}$ and Paolo Pellizzari ${ }^{(凶)}$ \\ Department of Economics, Ca' Foscari University, \\ Cannaregio 873, 30121 Venice, Italy \\ \{alessandro.moro, paolop\}@unive.it
}

\begin{abstract}
This paper presents an agent-based model of labor market participation, in which a population of agents is affected by adverse health shocks that impact the costs associated with work efforts, and decides whether to leave the labor market. This decision is simply taken by looking at the working behaviors of the other agents, comparing the respective levels of well-being and imitating the more advantageous decision of others. The analysis reveals that such mechanism of social learning suffices to replicate the existing empirical evidence regarding the decline in labor market participation of older people. As a consequence, the paper demonstrates that it is not necessary to assume perfect and unrealistic rationality at the individual level to reproduce a rational behavior in the aggregate.
\end{abstract}

Keywords: Labor market participation - Health shocks $\cdot$ Bounded rationality $\cdot$ Agent-based modeling

\section{Introduction}

This paper presents an agent-based model of labor market participation, in which a population of agents is naturally affected over time by adverse shocks that reduce their health and magnify the costs associated with work efforts. Agents decide whether or not to leave the labor market and retire (or access an otherwise available source of support, like a social security benefit). This decision is made by looking at the working behaviors of other randomly met agents and comparing the respective levels of well-being, rather than maximizing the utility function in a standard and fully rational way.

The analysis reveals that such mechanism of learning-by-meeting and imitation, even in the presence of otherwise entirely naive agents, can replicate some of the existing empirical evidence regarding the decline in labor market participation of older people. As a consequence, the paper shows that it is not necessary to assume full rationality at the individual level to reproduce a rational behavior in the aggregate.

The relevance of this claim can be understood by considering that the mainstream literature on labor supply and retirement choices is dominated by structural models, in which fully rational agents take decisions by maximizing their 
life-time utility. Examples of this kind of modeling approach, that also incorporates health shocks, are Bound et al. (2010), Burkhauser et al. (2004), French (2005), Gilleskie (1998), Gustman and Steinmeier (2002), Heyma (2004), Rust and Phelan (1997). To give some flavor, the agents in the paper by Rust and Phelan optimize their behavior working backward the optimal employment decision (i.e. how much to work) at any time $t$ between the age of 57 and 102 and choose whether to apply for Social Security. The situation faced by workers is described by 7 state variables, and optimality is determined in principle in each of 14,400 possible future occurrences maximizing the value of expected discounted utility. ${ }^{1}$

There is overwhelming evidence in experimental economics and psychology that shows how framing effects and contextual factors limit human rationality (Rabin 1998) and how poorly humans behave in problems that involve backward induction (Camerer 1997). Whether the previously described rational optimization can be performed by realistic agents is a no-brainer: consumer decision making is affected by information overload as soon as more than 10 options and 15 information items are provided (Lurie 2004), (Malhotra 1982), and it is well known that humans do not have good performances when dealing with (non trivial) probabilistic setups.

An alternative view, that incorporates the main findings of the empirical literature and much better accommodates with the psychological limitations of real agents, makes use of agent-based models where most often boundedly rational agents act using heuristics and cognitively sound adaptive rules of thumb. For instance, Lettau (1997) presents a model of portfolio decisions with agents that learn through a genetic algorithm how to behave based on random innovations and imitation. In particular, it is shown that investors in mutual funds exhibit the same patter of investments of the adaptive agents described in the paper and that the model closely matches the mutual fund data. Recently, Goudet et al. (2015) have developed an agent-based model with boundedly rational workers simulating the effects of suppression of fixed duration contracts in the French labor market.

More germane to our topic, Axtell and Epstein (1999) describe a model where a relatively small number of workers are rational, whereas a small proportion of them retire at random, and the vast majority just imitates the behaviors of the other agents in their social network. Again, high levels of optimal behavior can result in the aggregate despite low levels of individual rationality. Our paper supports a similar conclusion in a framework that also incorporates health shocks and proposes a different mechanism of social learning.

\footnotetext{
${ }^{1}$ This is an Herculean task requiring, among other things, to estimate transition probabilities from one state to all possible future states based on beliefs which, in turn, depends on 26 parameters to be determined. Other questionable assumptions include "rational expectations", a notion according to which all individual subjective probabilities equate the "objectively estimable population probability" and the capability to anticipate regulatory changes. The paper acknowledges several times that only some justification for such assumptions can be provided.
} 
It is worth stressing that the present paper proposes a mechanism of imitation of behaviors. This is the main difference with respect to the dispositional contagion framework proposed by Epstein (2014), in which each agent is not influenced by the actions of his peers, but by their dispositions. In our study the former approach is preferred because behaviors are more easily observed than dispositions.

The rest of the paper is organized as follows: the next section illustrates the main stylized facts that the model is able to reproduce, it describes the model, its calibration as well as the results of the simulations; finally, the last section concludes stressing the reasons why the present model can be considered a valid alternative to the mainstream approaches.

\section{Analysis}

This section of the paper is divided in three parts: the first one describes a few traits of the empirical evidence regarding the decline in the labor market participation of elderly workers affected by adverse health shocks; the second subsection describes in detail the proposed agent-based computational model; the last part shows the results of the model simulation and, in particular, how the model is able to capture some relevant stylized facts.

\subsection{Stylized Facts}

Data from the first wave of the English Longitudinal Study of Ageing (ELSA) are chosen in order to illustrate the empirical evidence regarding the labor market participation of elderly people and the main stylized facts that the model is able to replicate. This dataset contains information on both economic and labor supply variables, as well as on self-assessed health status.

The ELSA sample is representative of people aged 50 and over, living in private households in England. The interviews of the first wave began in March 2002 and spanned 12 months, completing in March 2003.

Figure 1 shows how labor market participation, measured as the proportion of people employed or self-employed, declines with the age of individuals. In particular, each curve is associated with a different level of health. ${ }^{2}$

Although labor market participation decreases with age in each health group, it is evident that people affected by poor health conditions have much lower participation rates than the individuals with a good health status, and this is true in every age group. In fact, people affected by adverse health shocks who are eligible to disability benefits or retirement have a strong incentive to leave the labor market. Issues related to the effects of various benefits are still debated by policymakers as they may artificially alter the participation to the labor market or offer awkward incentives to workers.

2 These statistics include people both in and outside the labor force. The data used to draw the curves in Fig. 1 are taken from the Table 4 A.3 in the Annex 4.1 - Tables on Work and Retirement of the first wave of the ELSA survey. http://www.elsa-project. ac.uk/reportWave1. 


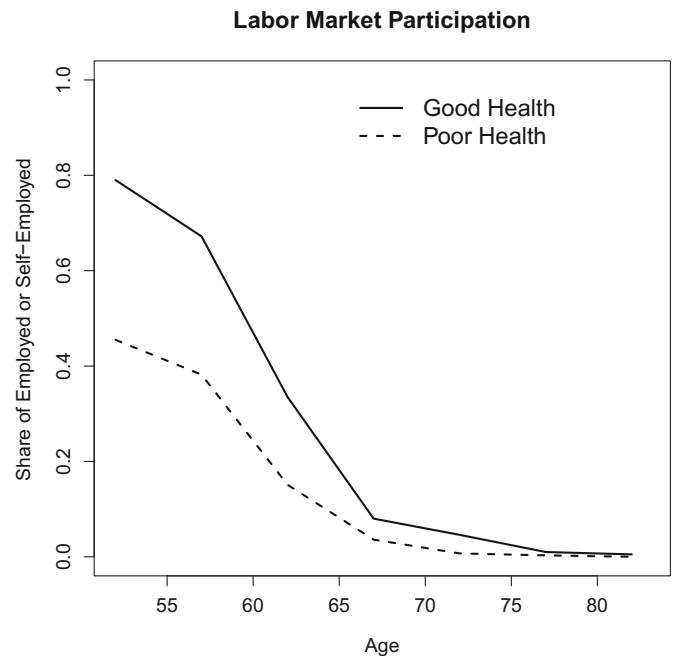

Fig. 1. Labor market participation by age and health. The solid line represents the participation of people with a good health, while the dashed line shows the participation of people affected by poor health conditions. Source: first wave of the English Longitudinal Study of Ageing.

\subsection{The Model}

In the model two types of agent, workers and pensioners, interact in a bidimensional torus space. At the beginning of the simulations $(t=0)$, the proportion of pensioners is equal to $p$. Workers receive a labor income $y^{l}$, which is greater than the pension $y^{p}$, but their work effort $e^{l}$ is bigger than the one of pensioners $e^{p} .^{3}$

Each agent $i$ starts with an age $a_{i, 0}$, randomly drawn from a uniform distribution on the $(50,89)$ interval, and receives a health $h_{i, 0}$, drawn from uniform distribution on the $\left(0, \frac{50}{a_{i, 0}}\right)$ interval.

Agents derive well-being $U_{i, t}^{s}$ from their working decisions that assumes the following expression:

$$
U_{i, t}^{s}=y_{i, t}^{s}-\frac{e_{i, t}^{s}}{h_{i, t}}
$$

where $s \in\{l, p\}$ denotes the state, labor or pension, of the individual. Thus, the well-being is an increasing function of income, whereas it decreases when the working effort increases. Moreover, the negative effect of exerting effort is greater when an agent suffers from a bad health, i.e. when $h_{i, t}$ is low.

\footnotetext{
${ }^{3}$ As mentioned previously, pensioners and pension income can also be interpreted as people who decide to leave the labor market and receive a subsidy or a disability benefit.
} 
This definition of well-being is consistent with the framework proposed by the OECD (2013). In particular, this institution measures well-being in terms of outcomes achieved in two broad domains: material living conditions (income and wealth, jobs and earnings, ...) and quality of life (physical and mental health, work-life balance, ....).

In each time step, an agent is randomly selected (asynchronous activation) and he moves to a random site of the bidimensional space, where he can meet another agent. The movement rule is very simple: the agent turns right at an angle that is randomly picked in $\{0,1,2, \ldots, 359\}$ degrees and moves forward $f$ patches (steps). If the moving agent "lands" on a patch hosting another person and the two are similar in terms of age and health status, they exchange information about their working choices, represented by the vector $(y, e)$, and their levels of well-being $U$. The similarity between the agents $i$ and $j$ is established if the following inequality holds:

$$
w \frac{\left|a_{i, t}-a_{j, t}\right|}{100}+(1-w)\left|h_{i, t}-h_{j, t}\right|<d,
$$

where $0 \leq w \leq 1$ measures the relative importance of age with respect to health, and $d$ represents a similarity threshold. A low (high) value of $w$ means that agents are more likely to imitate the behaviors of those with similar health conditions (age). Additionally, a higher value of the threshold $d$ implies that agents are more willing to copy the working choices of dissimilar players. The difference of ages is divided by 100 to obtain a measure, whose magnitude can be compared to that of the difference of health levels.

Once inequality (2) is satisfied (and only in this case), the two agents compare their respective well-being. Then, if $U_{i, t}^{s}<U_{j, t}^{s}$, agent $i$ will imitate the working choices $\left(y_{j, t}^{s}, e_{j, t}^{s}\right)$ of agent $j$, and vice versa. Social learning is therefore implemented with this kind of imitation mechanism involving no optimization or information processing on the part of the agents, who resemble walkers that randomly encounter other hikers and have a chance to discuss different experiences related to working satisfaction and opportunity to retire.

It is worth emphasizing that the movement in the bidimensional space can also be interpreted in metaphoric terms as the intensity of the social relationships of each agent: according to this view, a low value of the movement parameter $f$ means that agents are shy and more likely to often meet the same close peers and, accordingly, their social network is very limited; conversely, more mobile and lively agents have higher chances of stumbling upon different peers, their acquaintances' network is more widespread and can, on average, obtain more information about retirement decisions.

Quite naturally, age evolves deterministically over time $\left(a_{i, t+1}=a_{i, t}+1\right)$, while health is affected by random shocks over the life cycle:

$$
h_{i, t+1}=g h_{i, t}+(1-g) x_{i, t+1}
$$

where $0 \leq g \leq 1$ is a parameter capturing the persistence of health status, and $x_{i, t+1}$ is a random variable distributed with a uniform distribution on the 
$\left(0, \frac{50}{a_{i, t+1}}\right)$ interval: thus, the future level of health is a weighted average of the current health status and the random shock. This expression implies that an older agent is more likely to experience bad health shocks and, consequently, a decreasing level of health.

Finally, it is assumed that agents die at age 90 and they are replaced by new agents with an age equal to 50 and a health drawn from uniform distribution on the $\left(0, \frac{50}{a_{i, 0}}\right)$ interval. Moreover, these new agents are pensioners or workers with a probability equal to $p$ and $1-p$, respectively.

The model is implemented in NetLogo (Wilensky 1999). The user can choose the values for the model parameters, agents are randomly located in the bidimensional torus space, and each agent receives a random age, health and working condition. The code is available at http://tinyurl.com/paams16health and a screenshot of the Netlogo's graphical user interface is provided in Fig. 2.

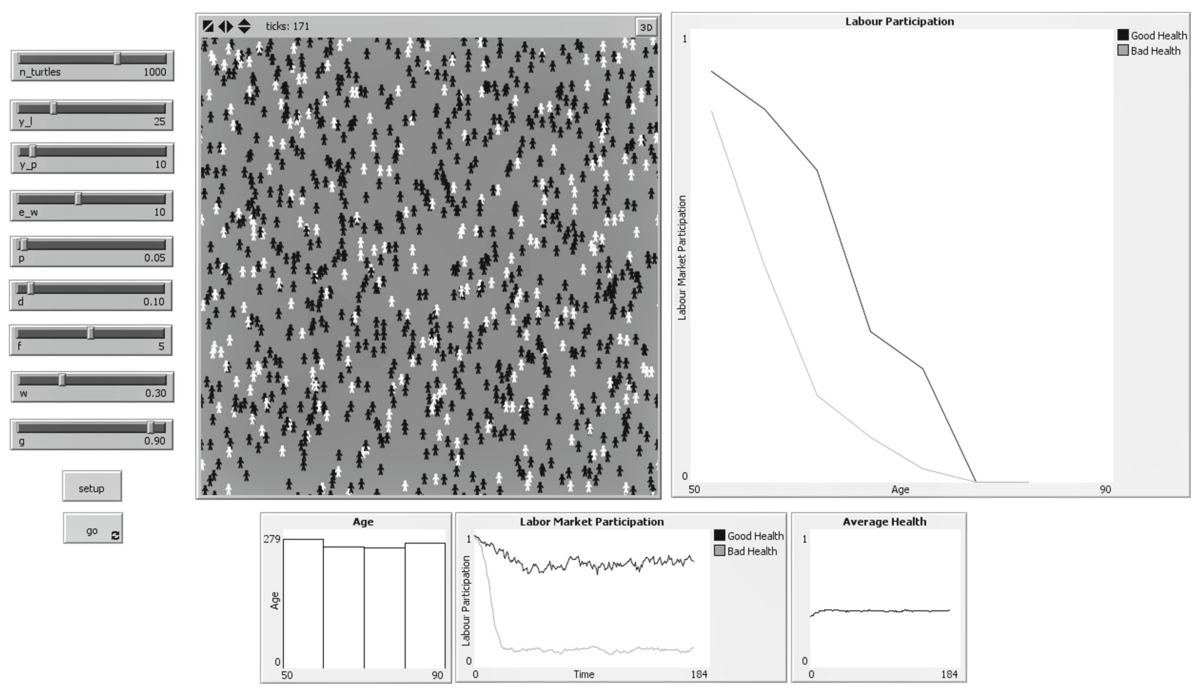

Fig. 2. Screenshot of Netlogo's graphical user interface.

In every time period the simulation evolves through the following steps:

1. an agent is randomly selected;

2. the chosen agent, following the movement rule, moves to a random site, where he/she may meet another agent;

3. if there is an encounter and the two agents are reasonably similar in terms of age and health conditions, the one with the lowest level of well-being will imitate the working choices of the agent with the highest well-being.

These simple rules are replicated for all agents. Then, once all agents have performed their tasks, the age and health status of each player is updated. 
Agents with an age equal to 90 "die", they are replaced by new ones endowed with a random health, time advances by one unit and the procedure starts again until the user quits the simulation or some specified time is reached. It is possible to monitor the evolution of the system by looking at some time series graphs that show the dynamics of the main model variables, as well as in the bidimensional space, in which workers and pensioners are depicted with different colors.

\subsection{Calibration and Results}

The model is simulated many times and under different conditions. On the one hand, it is assumed that the work effort of pensioners $e^{p}$ is equal to 0 and the benefits pensioners receive $y^{p}$ are kept constant and equal to 10: seen from the perspective of the workers, this means that the option of retirement is independent from health conditions and assumes a value of 10. The parameter $g$ is also constant and equal to 0.9 : this implies that the health status of agents resembles a persistent and slowly decaying $\mathrm{AR}(1)$ process, which appears a rather sensible assumption. On the other hand, the other parameters vary in the following sets: $y^{l} \in\{25,30,35\}, e^{l} \in\{5,10,15\}, w \in\{0.3,0.5,0.7\}, d \in\{0.1,0.2,0.3\}$, $f \in\{1,5,20\}$, and $p \in\{0.05,0.15,0.25\} .4$

For every combination of these values the model is simulated 100 times, for a total of 72,900 runs, and in each simulation the proportion of workers in the age range $[50,54],[55,59],[60,64],[65,69],[70,74],[75,79],[80,89]$ is recorded, ${ }^{5}$ distinguishing between agents with a good health, defined as those agents with $h_{i, t} \geq 0.5$, and the ones with a bad health, i.e. those with $h_{i, t}<0.5$. All simulations are halted after 200 time steps and the proportion of workers in the different age and health groups is evaluated at the end of the simulation. Then, for each combination of parameter values, the average proportion is calculated employing the respective 100 simulations, for each age range and health status.

The values of the parameters are selected in order to minimize the absolute distance between the (ELSA) observed labor market participation rates $l m p_{a, h}^{o b s}$, in the different age and health groups $(a, h)$, and those predicted by the model $l m p_{a, h}^{p r e d}$, divided by the product between the total number of age $A$ and health $H$ classes. Formally:

$$
D=\frac{1}{A H} \sum_{a, h}\left|l m p_{a, h}^{o b s}-\operatorname{lm} p_{a, h}^{p r e d}\right|
$$

The smallest value for $D$ is 0.036 , attained at the values listed in Table 1 . This means that, on average, the absolute deviation between observed and simulated labor market participation rates is about $3.6 \%$.

\footnotetext{
${ }^{4}$ Simulations are performed using the BehaviorSpace tool in NetLogo, which allows to specify the grid of parameter values and the number of simulations for each point of the grid.

${ }^{5}$ These seven classes of age correspond to the ones presented in the Table 4 A. 3 in the Annex 4.1 - Tables on Work and Retirement of the first wave of the ELSA survey.
} 
Table 1. Calibrated values of the model parameters.

\begin{tabular}{l|l|l}
\hline Parameter & Description & Value \\
\hline$p$ & Initial share of pensioners & 0.15 \\
\hline$y^{l}$ & Workers' income & 30 \\
\hline$y^{p}$ & Pensioners' income & 10 \\
\hline$e^{l}$ & Workers' effort & 15 \\
\hline$e^{p}$ & Pensioners' effort & 0 \\
\hline$f$ & Movement & 5 \\
\hline$w$ & Age weight & 0.7 \\
\hline$d$ & Similarity threshold & 0.1 \\
\hline$g$ & Health persistence & 0.9 \\
\hline
\end{tabular}

Figure 3 plots the labor market participation rates predicted by the model for the different age and health groups when the values contained in Table 1 are assigned to the parameters. Clearly, the model nicely fits the decreasing trend of the labor market participation curves with respect to age.

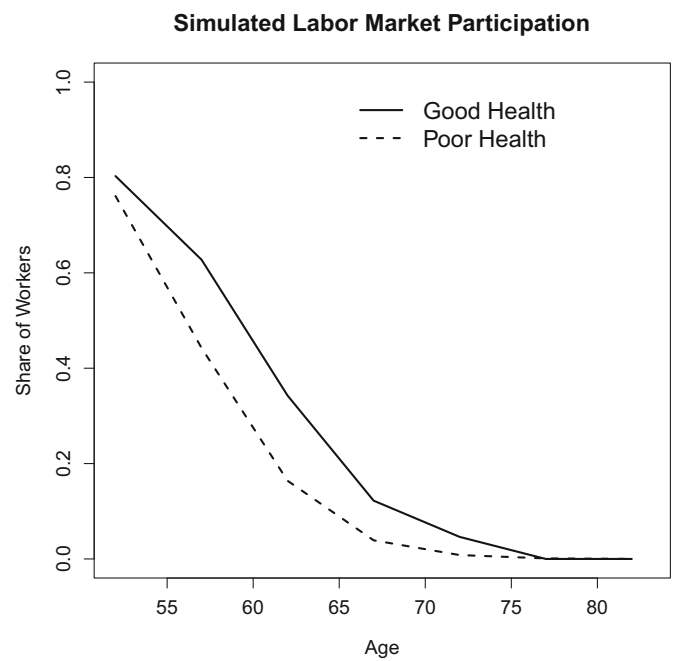

Fig. 3. Simulated labor market participation by age and health. The solid line represents the participation of agents with a good health $\left(h_{i, t} \geq 0.5\right)$, while the dashed line shows the participation of agents affected by poor health conditions $\left(h_{i, t}<0.5\right)$.

Real and simulated data are listed in Table 2, together with the absolute differences that build the error measure $D$. Values in the third row of the table and visual comparison of Figs. 1 and 3 show that most of modeling error is due to 
Table 2. Real and estimated labor market participation rates. The columns of the table in both health conditions correspond to the following age bins: [50, 54], [55, 59], $[60,64],[65,69],[70,74],[75,79],[80,89]$.

\begin{tabular}{l|l|l|l|l|l|l|l|l|l|l|l|l|l|l}
\hline Data & \multicolumn{4}{l|}{ Good health } & \multicolumn{1}{l|}{ Bad health } \\
\hline Real & 0.79 & 0.67 & 0.34 & 0.08 & 0.05 & 0.01 & 0.01 & 0.46 & 0.38 & 0.15 & 0.04 & 0.01 & 0.00 & 0.00 \\
\hline Simulated & 0.80 & 0.63 & 0.34 & 0.12 & 0.05 & 0.00 & 0.00 & 0.76 & 0.44 & 0.16 & 0.04 & 0.01 & 0.00 & 0.00 \\
\hline Abs. diff & 0.01 & 0.04 & 0.01 & 0.04 & 0.00 & 0.01 & 0.01 & $\mathbf{0 . 3 1}$ & 0.06 & 0.01 & 0.00 & 0.00 & 0.00 & 0.00 \\
\hline
\end{tabular}

a unique entry (0.31, boldfaced), the participation rate of workers aged $[50,54]$ with bad health. We lack additional information for that age/health group, but a more carefully chosen initial condition for the simulations may greatly reduce the mismatch.

Moreover, the participation of agents affected by adverse health shocks decreases more rapidly than that of agents with a good health. Both these aspects are consistent with the empirical evidence contained in the ELSA survey. In fact, given the functional form chosen for the well-being function, a worker with a bad health who meets a similar and already retired agent is more likely to change his status than a worker with a good health. Conversely, a retired agent with a good health may decide to enter again in the labor market when meeting a worker more easily than a pensioner with a bad health. This explains the differences in participation between agents with good and bad health conditions and the reason why labor market participation declines in both groups as health deteriorates due to the ageing of the population.

One of the main contributions of this paper is indeed the demonstration that no hyper-rational agent is needed in the model to replicate the existing evidence as it is sufficient to assume that simple agents randomly and repeatedly meet and mimic each other's behavior.

In order to illustrate the effects of changes in the values of the model parameters and prove the robustness of the obtained results, Fig. 4 compares the baseline calibration, reported in the first panel of the figure, with other scenarios characterized by some deviations from the values contained in Table 1.

Panel $4 \mathrm{~b}$ shows the effect of a higher income, i.e. $y^{l}$ equals 35 : it is evident that the labor market participation curves decline more slowly, especially for workers with a good health, because higher salaries make the outside option of retirement less attractive in relative terms. In fact, in an even more transparent way, the same pattern is observed in Panel $4 \mathrm{c}$, in which work effort $e^{l}$ is reduced to 10 (keeping income fixed at 30).

A lower value of the age weight $w$ means that agents are more likely to imitate the behaviors of those who are similar in terms of health conditions than the ones with the same age. This leads to an increase in the divergence of the labor market participation curves with respect to the baseline scenario, as shown in Panel 4d. A higher value of the similarity threshold is associated with steeper participation curves, as can be seen in Panel 4e, because workers tend to imitate the behaviors of more dissimilar agents that may have already decided to retire. 
(a) Baseline

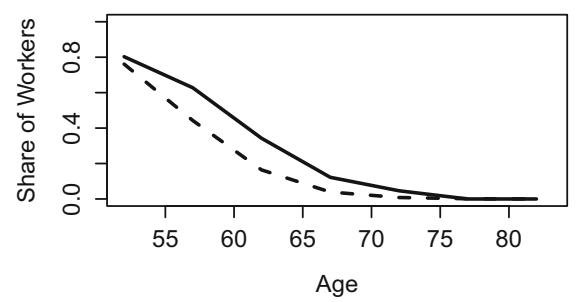

(c) Lower Effort

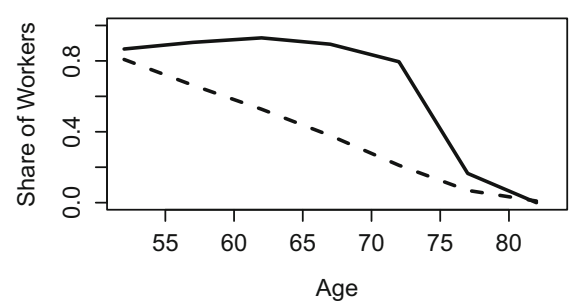

(e) Higher Threshold

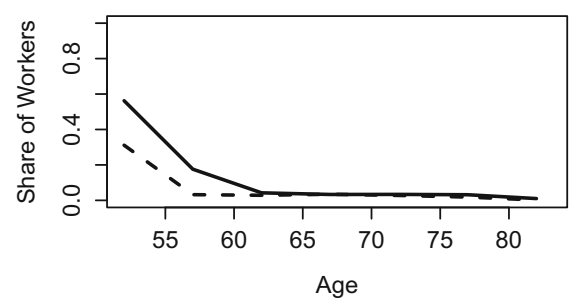

(b) Higher Income

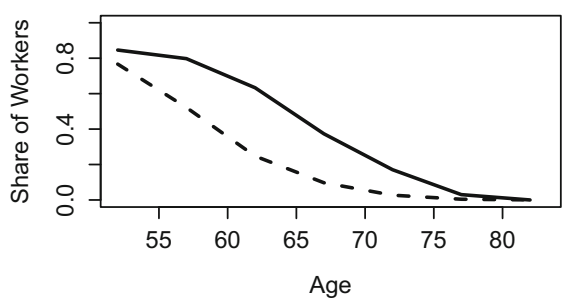

(d) Lower Age Weight

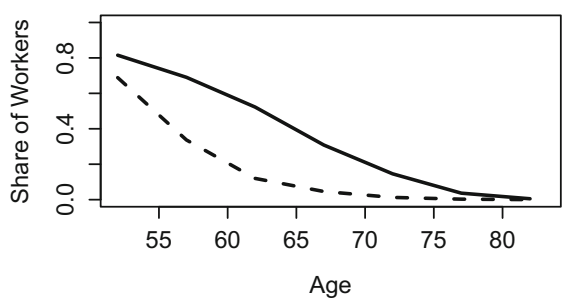

(f) Less Movement

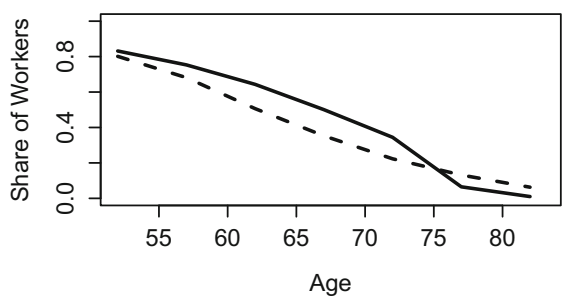

Fig. 4. Simulated labor market participation by age and health in different scenarios: (a) baseline calibration: values of Table 1 assigned to the model parameters; (b) higher labor income: $y^{l}=35$; (c) lower work efforts: $e^{l}=10$; (d) lower age weight: $w=0.3$; (e) higher threshold: $d=0.3$; (f) less movement: $f=1$. In each panel, the solid line represents the participation of agents with a good health $\left(h_{i, t} \geq 0.5\right)$, while the dashed line shows the participation of agents affected by poor health conditions $\left(h_{i, t}<0.5\right)$.

The last panel of the figure illustrates the effect of the mobility parameter $f$, showing that less mobile (or less socially connected) agents enjoy more infrequent meetings and, consequently, flatter labor market participation curves. Remarkably, nearly all of the graphs contained in Fig. 4 always preserve the same pattern, i.e. participation rates that decline when age increases, and in which workers affected by adverse health shocks are more likely to leave the labor market. The results of the model are therefore robust to different values of the parameters. 


\section{Conclusion}

The simple model described in this paper is able to replicate some relevant stylized facts contained in the ELSA survey, concerning the labor market participation of elderly people.

The core of the model is represented by naive agents moving in a bidimensional torus space, exchanging information on their work and health conditions, and making labor market choices according to this information: although extremely simple, the proposed mechanism of imitation and social learning is able to reproduce an aggregate behavior that can be considered rational and consistent with the empirical evidence, despite the bounded rationality of the individual agents.

It has already been stressed that the dynamics underlying the model can be interpreted in figurative terms: for instance, the movement in the bidimensional space is a measure of the intensity of the social relationships of each agent. Following this idea, agents that explore less the surrounding space are more likely to meet too often the same peers and fail to be faced with alternative and possibly better decisions regarding retirement. Conversely, more mobile agents have higher chances of seeing in their more widespread network diverse examples to imitate and improve their own well-being.

The model captures the most prominent stylized facts regarding the decline in labor market participation of aged workers, and well fits the participation curves with relatively few parameters. This parsimony is in sharp contrast with the mainstream structural models, which usually require numerous parameters to replicate the empirical evidence (John von Neumann famously stated that "With four parameters I can fit an elephant. And with five I can make him wiggle his trunk." Our model also would definitely be considered inadequate if you look at Table $1 \ldots$ )

Moreover, it is reasonable to think that more sophisticated forms of interaction between agents, such as the dispositional contagion mechanism assumed in the Epstein's (2014) notion of Agent_Zero, may probably deliver an outcome similar to our results.

In conclusion, all these considerations should allow to consider the model presented in this paper as a behaviorally plausible alternative to the standard modeling approaches that unrealistically assume the full rationality of individuals, despite an overwhelming psychological and experimental literature showing the limits of human reasoning.

\section{References}

Axtell, R.L., Epstein, J.M.: Coordination in transient social networks: an agent-based computational model of the timing of retirement. In: Aaron, H.J. (ed.) Behavioral Dimensions of Retirement Economics, pp. 161-183. Brookings Institution Press, Washington, DC (1999)

Bound, J., Stinebrickner, T.R., Waidmann, T.A.: Health, economic resources and the work decisions of older men. J. Econometrics 156, 106-129 (2010) 
Burkhauser, R.V., Butler, J.S., Gumus, G.: Dynamic programming model estimates of social security disability insurance application timing. J. Appl. Econometrics 19, 671-685 (2004)

Camerer, C.F.: Progress in behavioral game theory. J. Econ. Perspect. 11, 167-188 (1997)

Epstein, J.M.: Agent_Zero: Toward Neurocognitive Foundations for Generative Social Science. Princeton University Press, Princeton (2014)

French, E.: The effects of health, wealth, and wages on labour supply and retirement behaviour. Rev. Econ. Stud. 72, 395-427 (2005)

Gilleskie, D.B.: A dynamic stochastic model of medical care use and work absence. Econometrica 66, 1-45 (1998)

Goudet, O., Kant, J.D., Ballot, G.: Forbidding fixed duration contracts: unfolding the opposing consequences with a multi-agent model of the french labor market. In: Amblard, F., Miguel, F.J., Blanchet, A., Gaudou, B. (eds.) Advances in Artificial Economics. Lecture Notes in Economics and Mathematical Systems, vol. 676, pp. 151-167. Springer, Switzerland (2015)

Gustman, A.L., Steinmeier, T.L.: Retirement and the stock market bubble. NBER Working Paper No. 9404, National Bureau of Economic Research, Cambridge (2002)

Heyma, A.: A structural dynamic analysis of retirement behaviour in the Netherlands. J. Appl. Econometrics 19, 739-759 (2004)

Lettau, M.: Explaining the facts with adaptive agents: the case of mutual fund flows. J. Econ. Dyn. Control 21, 1117-1147 (1997)

Lurie, N.H.: Decision making in information-rich environments: the role of information structure. J. Consum. Res. 30, 473-486 (2004)

Malhotra, N.K.: Information load and consumer decision making. J. Consum. Res. 8, 419-430 (1982)

OECD: How's Life? 2013: Measuring Well-being. OECD Publishing, Paris (2013)

Rabin, M.: Psychology and economics. J. Econ. Lit. 36, 11-46 (1998)

Rust, J., Phelan, C.: How social security and medicare affect retirement behavior in a world of incomplete markets. Econometrica 65, 781-831 (1997)

Wilensky, U.: NetLogo. Center for Connected Learning and Computer-Based Modeling, Northwestern University, Evanston (1999). http://ccl.northwestern.edu/netlogo/ 